\title{
Onda Circumpolar Antártica: Influência na Variabilidade Climática do Estado do Rio Grande do Sul
}

\author{
Kyssyanne Samihra Santos Oliveira ${ }^{1}$, Maria Gertrudes Alvarez Justi da Silva ${ }^{2}$ \\ ${ }^{1}$ Departamento de Oceanografia e Ecologia, Centro de Ciências Humanas e Naturais, \\ Universidade Federal do Espírito Santo, Vitória, ES, Brasil. \\ ${ }^{2}$ Laboratório de Meteorologia, Universidade Estadual do Norte Fluminense Darcy Ribeiro, \\ Campos dos Goytacazes, RJ, Brasil.
}

Recebido: 27/1/2015 - Aceito: 13/12/2015

\begin{abstract}
Resumo
A influência das componentes interanuais (números de onda 2 - OCA2 e 3 - OCA3) da Onda Circumpolar Antártica sobre anomalias anuais regionais de precipitação do Rio Grande do Sul foi investigada utilizando-se dados meteoceanográficos que cobriram o período de 1981 a 2006. Os resultados encontrados aqui mostraram que enquanto existe um padrão claro de associação entre o comportamento da OCA3, ao longo do cinturão de latitude extratropical, e as anomalias anuais de precipitação nas quatro regiões homogêneas identificadas no RS, o mesmo não foi observado para a OCA2. Foi encontrado que anomalias de PNMM associadas a OCA3, no setor Atlântico Oeste do Oceano Sul, parecem ter um papel principal na modulação das anomalias anuais de precipitação no Rio Grande do Sul. Já as anomalias de TSM associadas a OCA3 parecem ter um papel secundário. Além disso, foi observada uma forte influência de ambas as componentes nas anomalias anuais de precipitação via anomalias anuais locais de umidade específica. Os padrões de associação entre as componentes OCA2 e OCA3 e as anomalias anuais de precipitação encontrados aqui fornecem informações importantes tanto para o entendimento do clima no Estado, como para o aprimoramento da previsão climática para o mesmo.
\end{abstract}

Palavras-chave: Onda Circumpolar Antártica, Rio Grande do Sul, anomalias anuais de precipitação.

\section{Antarctic Circumpolar Wave: Influence in the Climate Variability of the Rio Grande do Sul State}

\begin{abstract}
The influence of interannual components (wavenumbers 2 - OCA2 and 3 - OCA3) of the Antarctic Circumpolar Wave on regional annual precipitation anomalies of Rio Grande do Sul was investigated using meteoceanographic data covered the period from 1981 to 2006 . The results found here showed that while there is a clearest pattern of association between the behavior of OCA3 along the extratropical latitude belt and annual precipitation anomalies in the four homogeneous regions identified in the RS, the same was not observed for OCA2. It was found that SLP anomalies associated with OCA 3 on the west sector of the South Atlantic Ocean, appear to have a major role in the modulation of annual precipitation anomalies in Rio Grande do Sul. On the other hand, the SST anomalies associated with OCA3 seem to have a secondary role. Moreover, was observed a strong influence of both components on annual precipitation anomalies by local annual anomalies of specific humidity. The patterns of association between the behavior of OCA2 and OCA 3 components and the annual precipitation anomalies founded here provide important informations to both the understanding of the climate in the state and for the improvement of climate forecasts for the RS.
\end{abstract}

Keywords: Antarctic Circumpolar Wave, Rio Grande do Sul, annual anomalies of precipitation.

Autor de correspondência: Kyssyanne Samihra Santos Oliveira, kyssyanne.samihra@gmail.com. 


\section{Introdução}

O conhecimento da variabilidade climática tem se mostrado de fundamental importância para diversos setores da economia, com destaque para o setor agropecuário devido a sua dependência direta com a disponibilidade hídrica. Um estado que se destaca por sua produção agropecuária é o Rio Grande do Sul, e apesar da relevância deste setor para a economia do estado, sabe-se que a variabilidade anual dos rendimentos é muito ampla, atingindo muitas vezes níveis desastrosos da safra agrícola (Berlato e Cordeiro, 2005). Diversos estudos (Berlato e Fontana, 2003; Fontana e Berlato, 2005) têm mostrado que a variabilidade climática, especialmente a variabilidade interanual da precipitação, é a principal causa da variabilidade dos rendimentos e da produção agrícola no Estado.

Situado no sudeste da América do Sul, o estado do Rio Grande do Sul está sujeito à atuação de forçantes climáticas de diversas escalas espaço-temporais, tais como os padrões de variabilidade climática El-Niño - Oscilação Sul, Oscilação Antártica e Oscilação Decadal do Pacífico (Britto et al., 2008; Prestes et al., 2010). O estudo da influência de padrões de variabilidade climática provenientes das médias e altas latitudes do Hemisfério Sul na variabilidade climática do estado do Rio Grande do Sul ainda é recente, de forma que alguns padrões praticamente não tiveram sua influência estudada. Um exemplo é a Onda Circumpolar Antártica (OCA), um padrão climático de grande escala espaço-temporal, inerente às médias e altas latitudes do Hemisfério Sul, e caracterizado por apresentar anomalias positivas (negativas) de temperatura da superfície do mar associadas com ventos meridionais de norte (de sul), e com retração (expansão) da cobertura de gelo marinho ao redor da Antártica (White e Peterson, 1996). Os processos responsáveis pela geração da Onda Circumpolar Antártica foram investigados por trabalhos como os de Christoph et al. (1998), Venegas (2003) e Connolley (2003). Esses estudos encontraram que na escala de tempo interanual, a OCA é a soma de dois modos, um com número de onda dois e o outro com número de onda três e que o modo El-niño/Oscilação Sul e o acoplamento oceanoatmosfera extratropical são os mecanismos responsáveis por sua geração.

Diferentemente de outros padrões de variabilidade climática, como El-niño - Oscilação Sul e a Oscilação Antártica, a Onda Circumpolar Antártica não possui um índice atualizado que demonstre sua variabilidade ao longo do tempo. Embora Bian e Lin (2012) tenham construído um índice para a Onda Circumpolar Antártica considerando o período de 1957 a 2010, os autores só consideraram a componente atmosférica do padrão, negligenciando a componente oceânica. Além disso, os autores não consideraram as diferentes componentes interanuais do padrão já previamente identificadas em outros trabalhos (Connolley (2003); Venegas, 2003).
O estudo da influência da Onda Circumpolar Antártica no clima é de caráter consideravelmente recente, mas já tem fornecido importantes resultados. Estudos como o de White e Cherry (1999) na Nova Zelândia, White (2000) na Austrália e Prabhu et al. (2010) na Índia encontraram uma forte influência da Onda Circumpolar Antártica sobre a precipitação nesses países e concluíram que considerar esta influência pode representar um importante avanço na previsão climática destes locais. Khan et al. (1998) foram os primeiros a encontrar uma forte relação entre a precipitação na Região Sul do Brasil e o cinturão de temperatura da superfície do mar do Oceano Sul, onde se situa a Frente Circumpolar Antártica. Com base nos seus resultados, os autores sugeriram que as anomalias das precipitações no sul do Brasil estão significativamente correlacionadas com as anomalias climáticas provocadas pela Onda Circumpolar Antártica.

Diante da lacuna de conhecimento sobre a influência da Onda Circumpolar Antártica nas anomalias anuais de precipitação no estado do Rio Grande do Sul, a proposta deste estudo é investigar o comportamento da Onda Circumpolar Antártica em anos com anomalias positivas e negativas de precipitação e encontrar as relações entre este comportamento e essas anomalias. Desde que não há um índice atualizado que demonstre a variabilidade temporal da Onda Circumpolar Antártica, a evolução temporal desse modo para o período de 1981 a 2006 também é estudada.

\section{Materiais e Métodos}

\subsection{Material}

Neste estudo foram utilizados dois conjuntos de dados, ambos cobrindo o período de 1981 a 2006. O primeiro conjunto consiste de valores totais mensais de precipitação referentes a 25 estações meteorológicas distribuídas no Estado do Rio Grande do Sul (Tabela 1). Estes dados foram obtidos do Banco de Dados do Centro de Meteorologia Aplicada pertencente à Fundação Estadual de Pesquisa Agropecuária - FEPAGRO/SCT-RS e do $8^{\circ}$ Distrito de Meteorologia do Instituto Nacional de Meteorologia INMET. Os dados de precipitação utilizados neste trabalho tiverem suas falhas preenchidas por Diniz (2002), que utilizou o método das correlações para obter séries completas.

$\mathrm{O}$ segundo conjunto de dados refere-se às médias mensais de temperatura da superfície do mar (TSM), pressão ao nível médio do mar (PNMM), umidade específica (UMI) em $1000 \mathrm{hPa}$ e componente meridional do vento (VMS) em $1000 \mathrm{hPa}$. Estes dados foram extraídos a partir da nova reanálise do NCEP - Climate Forecast System Reanalysis (CFSR; Saha et al., 2010). A escolha da CFSR como fonte de dados decorre de algumas das vantagens que esta reanálise possui em relação às anteriores, tais como um sistema de modelagem aprimorado com maior resolução e um sistema de assimilação de dados oceânicos e atmosféricos parcialmente acoplado (Saha et al., 2010). Além 
Tabela 1 - Estações Meteorológicas do Estado do Rio Grande do Sul, coordenadas geográficas, altitude e instituições a que pertencem.

\begin{tabular}{|c|c|c|c|c|}
\hline Estação & Latitude & Longitude & Altitude (m) & Fonte \\
\hline Bagé & $31^{\circ} 21^{\prime}$ & $54^{\circ} 06^{\prime}$ & 215 & $8^{\circ}$ DISME \\
\hline Bento Gonçalves & $29^{\circ} 15^{\prime}$ & $51^{\circ} 31^{\prime}$ & 619 & $8^{\circ}$ DISME \\
\hline Bom Jesus & $28^{\circ} 40^{\prime}$ & $50^{\circ} 26^{\prime}$ & 1047 & $8^{\circ}$ DISME \\
\hline Cachoeira do Sul & $30^{\circ} 02^{\prime}$ & $52^{\circ} 53^{\prime}$ & 72 & $8^{\circ}$ DISME \\
\hline Caxias do Sul & $29^{\circ} 10^{\prime}$ & $51^{\circ} 12^{\prime}$ & 785 & $8^{\circ}$ DISME \\
\hline Cruz Alta & $28^{\circ} 38^{\prime}$ & $53^{\circ} 36^{\prime}$ & 472 & $8^{\circ}$ DISME \\
\hline Encruzilhada do Sul & $30^{\circ} 32^{\prime}$ & $52^{\circ} 31^{\prime}$ & 427 & $8^{\circ}$ DISME \\
\hline Ijuí & $28^{\circ} 23^{\prime}$ & $53^{\circ} 54^{\prime}$ & 448 & FEPAGRO \\
\hline Iraí & $27^{\circ} 11^{\prime}$ & $53^{\circ} 14^{\prime}$ & 222 & $8^{\circ}$ DISME \\
\hline Lagoa Vermelha & $28^{\circ} 25^{\prime}$ & $51^{\circ} 35^{\prime}$ & 836 & $8^{\circ}$ DISME \\
\hline Passo Fundo & $28^{\circ} 15^{\prime}$ & $52^{\circ} 21^{\prime}$ & 676 & $8^{\circ}$ DISME \\
\hline Pelotas & $31^{\circ} 52^{\prime}$ & $52^{\circ} 21^{\prime}$ & 13 & $8^{\circ}$ DISME \\
\hline Porto Alegre & $30^{\circ} 01^{\prime}$ & $51^{\circ} 13^{\prime}$ & 46 & $8^{\circ}$ DISME \\
\hline Rio Grande & $32^{\circ} 01^{\prime}$ & $52^{\circ} 05^{\prime}$ & 5 & $8^{\circ}$ DISME \\
\hline Santa Maria & $29^{\circ} 42^{\prime}$ & $53^{\circ} 42^{\prime}$ & 95 & $8^{\circ}$ DISME \\
\hline Santa Rosa & $27^{\circ} 51^{\prime}$ & $54^{\circ} 25^{\prime}$ & 360 & $8^{\circ}$ DISME \\
\hline Santa Vitória do Palmar & $33^{\circ} 31^{\prime}$ & $53^{\circ} 21^{\prime}$ & 6 & $8^{\circ}$ DISME \\
\hline Santana do Livramento & $30^{\circ} 53^{\prime}$ & $55^{\circ} 32^{\prime}$ & 210 & $8^{\circ}$ DISME \\
\hline São Borja & $28^{\circ} 39^{\prime}$ & $56^{\circ} 00^{\prime}$ & 96 & FEPAGRO \\
\hline São Gabriel & $30^{\circ} 20^{\prime}$ & $54^{\circ} 19^{\prime}$ & 124 & $8^{\circ}$ DISME \\
\hline São Luiz Gonzaga & $28^{\circ} 23^{\prime}$ & $54^{\circ} 58^{\prime}$ & 254 & $8^{\circ}$ DISME \\
\hline Taquari & $29^{\circ} 48^{\prime}$ & $51^{\circ} 49^{\prime}$ & 76 & $8^{\circ}$ DISME \\
\hline Torres & $29^{\circ} 20^{\prime}$ & $49^{\circ} 43^{\prime}$ & 43 & $8^{\circ}$ DISME \\
\hline Uruguaiana & $29^{\circ} 45^{\prime}$ & $57^{\circ} 05^{\prime}$ & 74 & $8^{\circ}$ DISME \\
\hline Veranópolis & $28^{\circ} 56^{\prime}$ & $51^{\circ} 33^{\prime}$ & 705 & FEPAGRO \\
\hline
\end{tabular}

disso, a CFSR inclui um modelo de gelo-marinho interativo e um modelo atmosférico que contém variações observadas de dióxido de carbono sobre todo o período de 1979-2009, juntamente com mudanças em aerossóis e outros gases traços, além de variações na atividade solar (Saha et al., 2010).

\subsection{Métodos}

2.2.1. Cálculo das anomalias anuais de precipitação e identificação das regiões homogêneas

Para cada uma das 25 estações meteorológicas foram calculadas médias anuais de precipitação, e a partir dessas, foi feito o cálculo das anomalias anuais normalizadas. Uma Análise de Agrupamento, utilizando-se o método de Ward, foi realizada a fim de se identificar regiões homogêneas de anomalias anuais normalizadas de precipitação. Esta abordagem foi escolhida, pois possibilita a identificação de estações com comportamento semelhante na escala interanual, e assim permite a detecção de diferentes modos de influência dos padrões de variabilidade climática sobre o clima do estado do Rio Grande do Sul. Neste estudo, a medida de dissimilaridade utilizada foi a distância euclidia- na. O método de Ward foi escolhido por ser um método hierárquico que tem sido usado com sucesso em diversos trabalhos de identificações de regiões homogêneas de precipitação ou temperatura (Fechine e Galvíncio, 2008; Guedes et al., 2010).

Identificadas as regiões homogêneas, uma nova série de anomalias foi calculada, a partir da média aritmética das anomalias anuais das estações pertencentes a cada região homogênea. Como resultado obteve-se 26 anomalias médias regionais (1981 a 2006) para cada região homogênea

\subsubsection{Identificação e evolução espaço-temporal da Onda Circumpolar Antártica}

A técnica utilizada para a identificação e reconstrução da evolução espaço-temporal das componentes na banda interanual de número de onda 2 e número de onda 3 da Onda Circumpolar Antártica foi a Análise Espectral pelo Método das Janelas Múltiplas ("Multitaper") associada à Decomposição dos Valores Singulares (MJM-DVS) (Mann e Park, 1999). Esta técnica já tem sido utilizada em outros estudos para estudar a Onda Circumpolar Antártica (Mann e Park, 1999; Venegas, 2003; White et al., 2004). Tais estudos encontraram na banda interanual duas componen- 
tes da Onda Circumpolar Antártica: a OCA2 (número de onda 2) e a OCA3 (número de onda 3).

Para a identificação da OCA, a região selecionada compreende o cinturão de latitude entre $30^{\circ} \mathrm{S} \mathrm{e} 90^{\circ} \mathrm{S}$. Este cinturão de latitude foi selecionado, pois segundo White e Annis (2004) e White et al. (2004), a Onda Circumpolar Antártica pode ser encontrada propagando-se ao redor do globo desde os subtrópicos até os extratrópicos. Além disso, a resolução espacial dos dados de TSM, PNMM, UMI e VMS, que era inicialmente de $0.5^{\circ}$ de latitude por $0.5^{\circ}$ de longitude, foi reduzida para $5^{\circ}$ de latitude por $5^{\circ}$ de longitude por meio do método "Box Averaging". A redução da resolução espacial foi realizada devido a escala espaçotemporal necessária para detectar a variabilidade climática em escala de bacia sobre o oceano superior global (White, 1995). Primeiramente foi feita uma análise MJM-DVS conjunta entre as anomalias normalizadas de TSM e PNMM a fim de identificar e isolar as duas componentes OCA2 e OCA3. Uma segunda análise MJM-DVS conjunta foi feita entre as anomalias normalizadas das quatro variáveis: TSM, PNMM, UMI e VMS a fim de isolar os padrões de vento meridional e de umidade específica associados às componentes OCA2 e OCA3.

\section{Resultados e Discussão}

\subsection{Regiões homogêneas das anomalias anuais de precipitação do Estado do Rio Grande do Sul (RS)}

A partir da Análise de Agrupamento das anomalias anuais regionais de precipitação foi possível a identificação de quatro regiões homogêneas (Fig. 1). Como já observado em outros trabalhos (Araújo (2005); Machado et al. (2010) e Santos et al. (2011)), a aproximação física das estações meteorológicas não garantiu a afinidade climática entre elas e as regiões homogêneas parecem se agrupar pela combinação da atuação de forçantes locais (variação latitudinal, relevo, vegetação e atuação de massas de ar) e de forçantes remotas (padrões de variabilidade climática).

Uma análise da variabilidade interanual das anomalias anuais regionais de precipitação foi realizada através do cálculo da raiz quadrada da média (RMS) dos valores das séries de anomalias de cada uma das regiões homogêneas, a fim de mostrar onde a variabilidade interanual é relativamente fraca ou forte. A partir da Tabela 2, pode ser notado que dentre as regiões homogêneas das anomalias anuais regionais de precipitação, as porções Central e Noroeste (Regiões 2 e 4) do RS foram as regiões em que foram observadas as maiores diferenças de precipitação de um

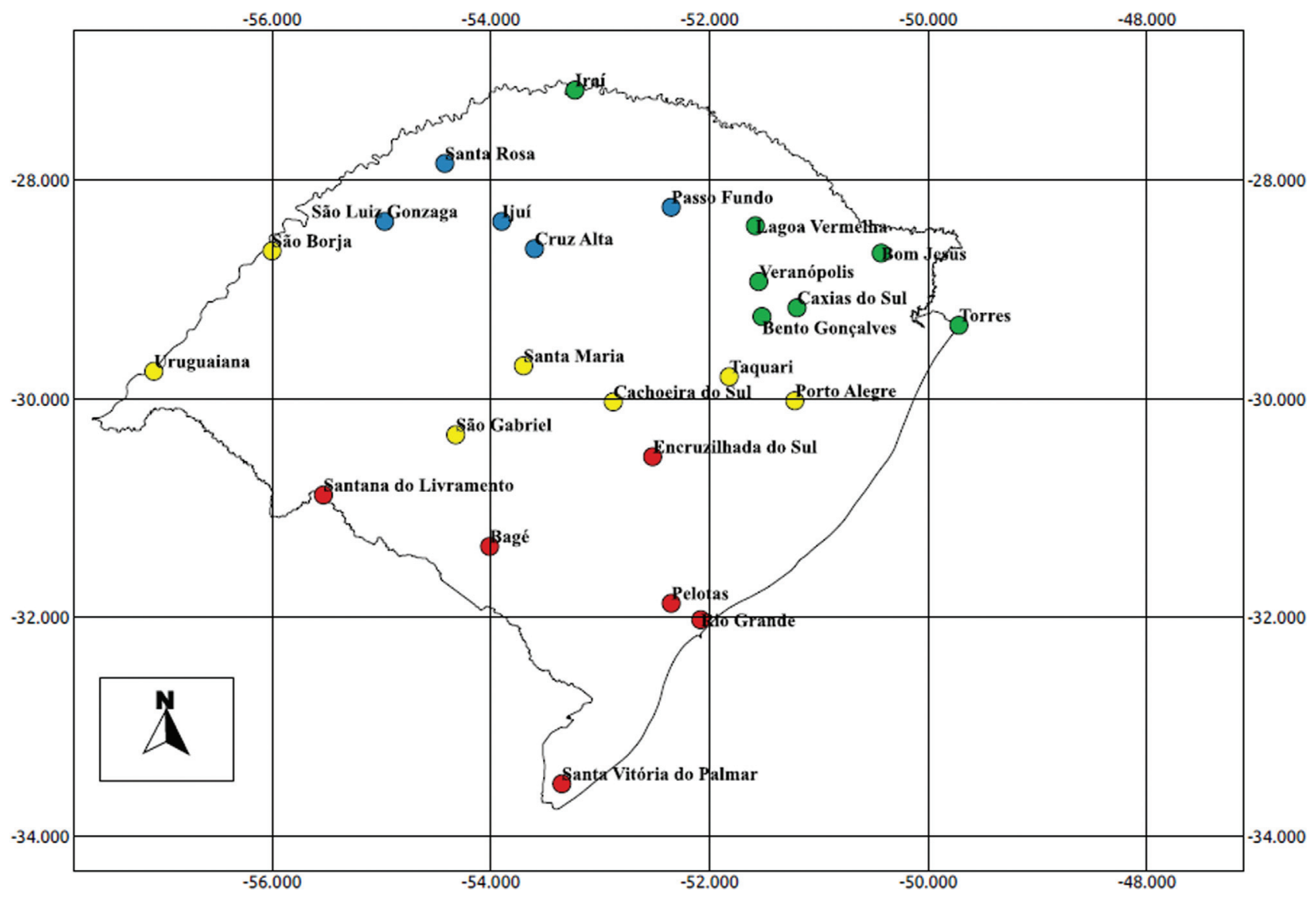

Figura 1 - Regiões homogêneas das anomalias anuais de precipitação, para o período de 1981 a 2006 . As estações meteorológicas pertencentes às Regiões homogêneas 1, 2, 3 e 4 são sinalizadas, respectivamente, pelos círculos de cores vermelha, amarela, verde e azul. 
Tabela 2 - Raiz média quadrada das anomalias anuais regionais de precipitação para o período de 1981 a 2006.

\begin{tabular}{ll}
\hline Regiões Homogêneas & RMS \\
\hline 1 & 0,840 \\
2 & 0,879 \\
3 & 0,876 \\
4 & 0,932 \\
\hline
\end{tabular}

ano para outro, indicando assim que são estas as regiões do RS que mais estão sob a influência das forçantes climáticas que atuam na escala interanual. Tal comportamento pode estar associado a influência diferenciada das forçantes climáticas sobre os diversos sistemas meteorológicos, que atuam sobre o Rio Grande do Sul, ao longo do ano, e consequentemente sobre o padrão espaço-temporal de precipitação derivado da ação destes sobre as diferentes regiões homogêneas (Britto et al., 2006). Os autores encontraram que sazonalmente, no litoral e no centro-sul do Estado, os meses de inverno são os mais chuvosos, devido a freqüência de passagens dos sistemas frontais. Já no sudoeste do Estado, o outono é mais chuvoso, devido a atuação dos bloqueios atmosféricos. No noroeste do RS, os autores encontraram que os meses da primavera são os mais chuvosos, devido a formação dos Complexos Convectivos de Mesoescala. E para a região nordeste, o verão é mais chuvoso, em função das chuvas convectivas associadas a sistemas atmosféricos como a Zona de Convergência do Atlântico Sul.

\subsection{Evolução espaço-temporal da Onda Circumpolar Antártica}

O espectro de Variância Fracional Local do campo conjunto de TSM e PNMM mostrado na Fig. 2 revelou as componentes da Onda Circumpolar Antártica com número de onda 2 (OCA2) e número de onda 3 (OCA3) com

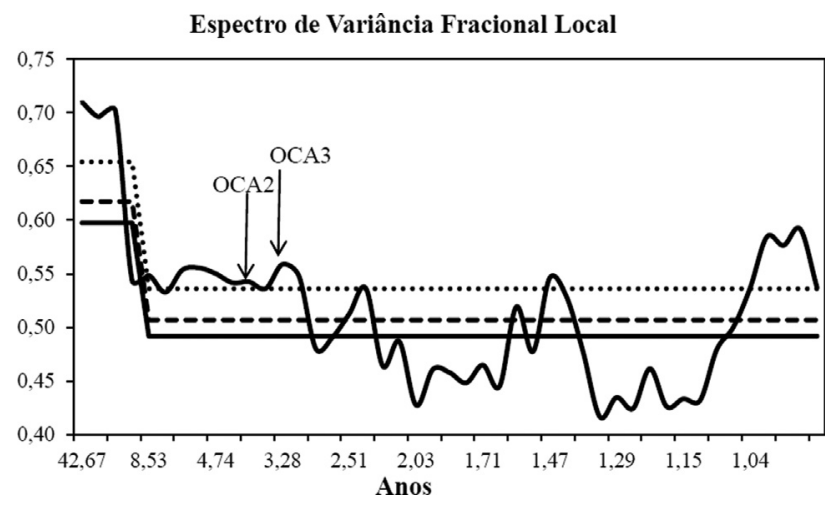

Figura 2 - Espectro de Variância Fracional Local do campo conjunto de TSM e PNMM baseado no período de 1981-2006, com níveis de significância de 99\% (linha pontilhada), 95\% (linha tracejada) e 90\% (linha contínua) calculados através da técnica de reamostragem de bootstrap. período de 4,27 anos e 3,55 anos, respectivamente. Os picos foram encontrados com significância de $99 \%$.

A identificação das componentes OCA2 e OCA3 foi feita comparando-se os padrões espaço-temporal reconstruídos de anomalias de TSM e PNMM de cada um dos picos significativos detectados na escala interanual neste estudo, com os padrões espaço-temporal das componentes de número de onda 2 e 3 já identificados por Venegas (2003) e White e Annis (2004). A reconstrução dos padrões mostrou anomalias de TSM associadas as OCA2 (Fig. 3) e OCA3 (Fig. 4) propagando-se para leste ao redor do Oceano Sul com as maiores anomalias localizadas no Oceano Pacífico. Em ambas as componentes essas anomalias positivas de TSM foram observadas a leste de centros de anomalias positivas de PNMM. Tais resultados concordam com os padrões espaciais associados às componentes já identificados em outros trabalhos (White e Peterson, 1996; Connolley, 2003; Venegas, 2003; White e Annis, 2004).

A segunda análise conjunta de MJM-DVS, entre as anomalias de TSM, PNMM, UMI e VMS, mostrou que para a banda de latitude entre $40^{\circ} \mathrm{S}$ e $60^{\circ} \mathrm{S}$ (banda latitudinal referente a principal trajetória da OCA (White e Chen, 2002; White e Annis, 2004)), a OCA2 apresentou anomalias positivas de PNMM (Fig. 3b) associadas a anomalias positivas de UMI (Fig. 5b) para os setores Pacífico, Índico e Atlântico Central e Leste do Oceano Sul e anomalias positivas de PNMM associadas a anomalias negativas de UMI para o setor Atlântico Oeste do Oceano Sul.

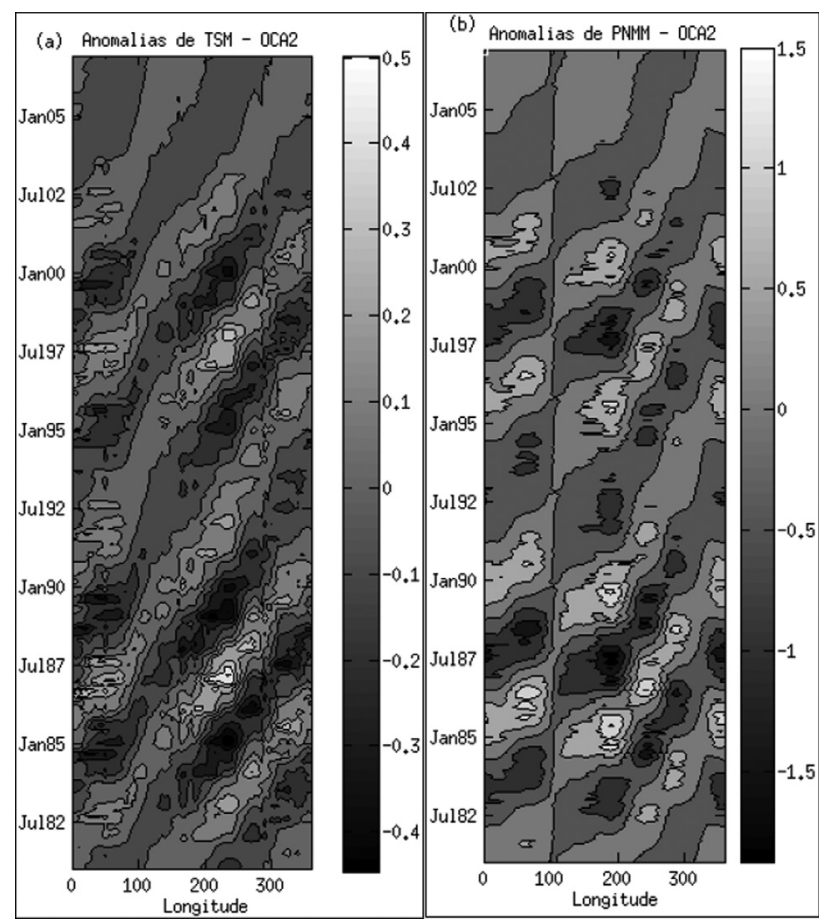

Figura 3 - Diagrama de Hovmöller obtido a partir da média zonal da banda de latitude entre $40^{\circ} \mathrm{S}$ e $60^{\circ} \mathrm{S}$, trajetória principal da OCA, das anomalias normalizadas de TSM (a) e PNMM (b) associadas a OCA2. Tons claros representam anomalias positivas e tons escuros representam anomalias negativas. 


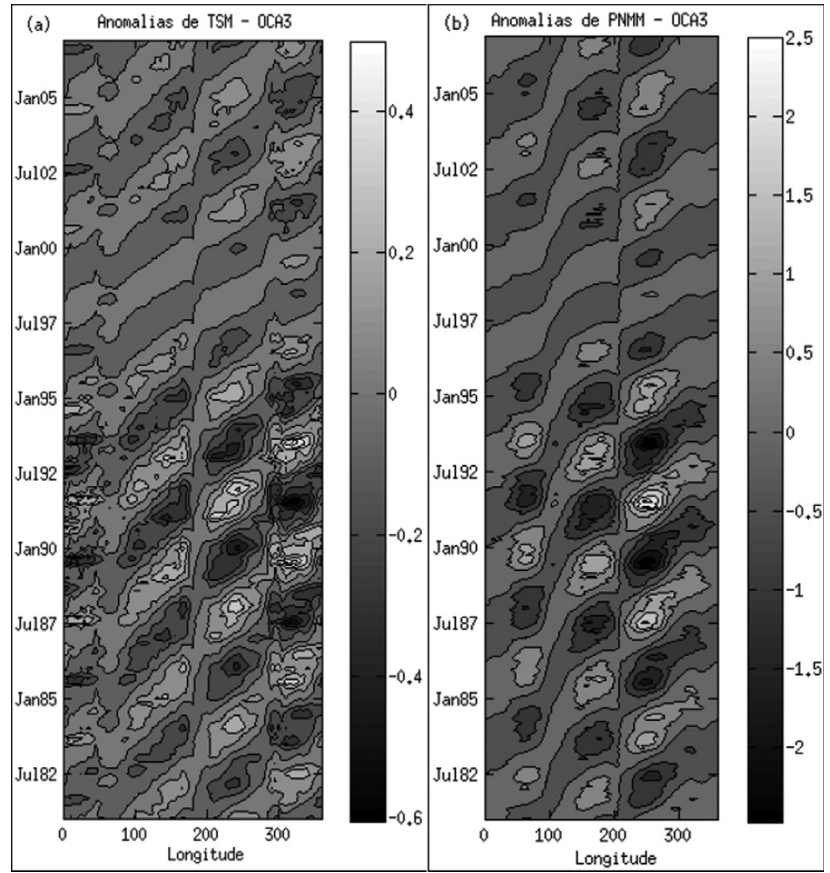

Figura 4 - Diagrama de Hovmöller obtido a partir da média zonal da banda de latitude entre $40^{\circ} \mathrm{S}$ e $60^{\circ} \mathrm{S}$, trajetória principal da OCA, das anomalias normalizadas de TSM (a) e PNMM (b) associadas a OCA3. Tons claros representam anomalias positivas e tons escuros representam anomalias negativas.
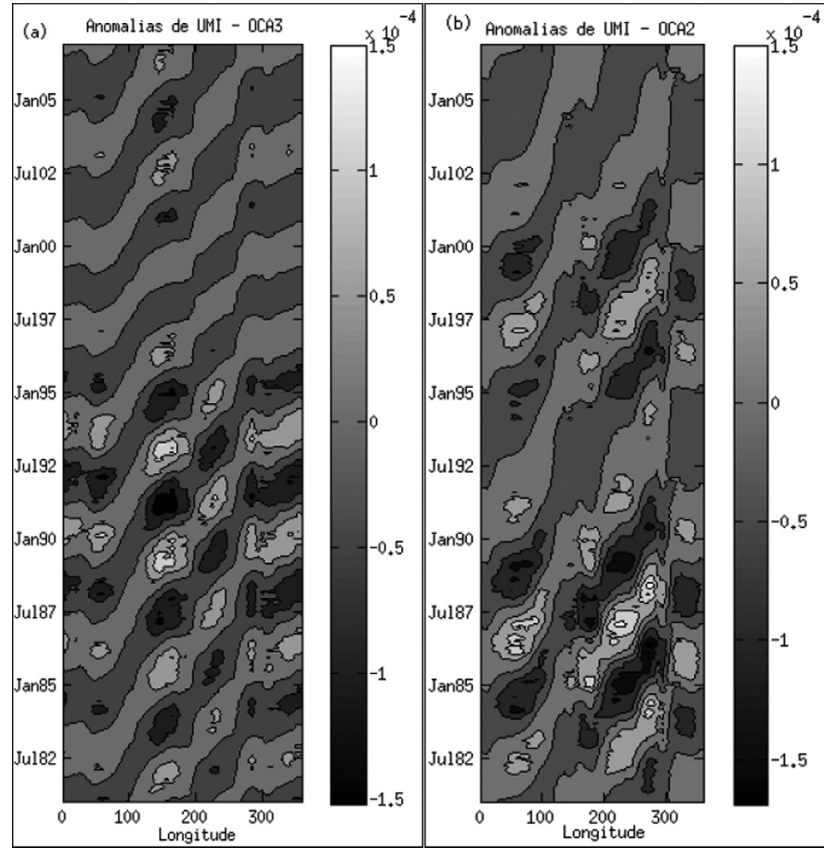

Figura 5 - Diagrama de Hovmöller obtido a partir da média zonal da banda de latitude entre $40^{\circ} \mathrm{S}$ e $60^{\circ} \mathrm{S}$, trajetória principal da OCA, das anomalias normalizadas de UMI associadas a OCA3(a) e OCA2(b). Tons claros representam anomalias positivas e tons escuros representam anomalias negativas.
Já a OCA3 apresentou anomalias positivas de PNMM (Fig. 4b) associadas a anomalias positivas de UMI (Fig. 5a) para os setores Índico e Pacífico Oeste e Central do Oceano Sul e anomalias positivas de PNMM associadas a anomalias negativas de UMI para os setores Pacífico Leste, Atlântico Oeste, Central e Leste do Oceano Sul. Isto indica que ocorre uma mudança no comportamento do acoplamento do sistema oceano-atmosfera quando a OCA propaga-se através do setor Pacífico Central para os setores Pacífico Leste e Atlântico Oeste do Oceano Sul. Esta mudança de comportamento quando a OCA propaga-se para leste entre esses setores também foi encontrada por White e Chen (2002). Estes autores encontraram, ao longo da trajetória da OCA, correlações cruzadas positivas entre anomalias de TSM, calor sensível mais latente, calor latente e precipitação, ao longo dos setores Atlântico Leste, Índico, e Pacífico Central e Oeste do Oceano Sul. Por outro lado, nos setores Pacífico Leste e Atlântico Oeste do Oceano Sul, correlações negativas entre as anomalias de TSM, calor sensível mais latente, calor latente e precipitação. Além disso, White e Chen (2002) encontraram anomalias positivas de PNMM deslocadas aproximadamente $45^{\circ}$ para leste das anomalias positivas de TSM, indicando uma invasão do centro de anomalia positiva de PNMM sobre o centro de anomalia positiva de TSM nos setores Pacífico Leste e Atlântico Oeste do Oceano Sul.

Tal aproximação entre os centros de anomalias positivas de TSM e PNMM quando se aproximam da Passagem de Drake, também foi observada no presente estudo (Figs. 3 e 4). Com base nestes resultados, White e Chen (2002) sugeriram que, nos setores Pacífico Leste e Atlântico Oeste do Oceano Sul, a influência da anomalia positiva de TSM na anomalia de calor latente é suprimida pela influência do centro de anomalia positiva de PNMM de núcleo quente, resultando nas anomalias negativas de UMI encontradas neste estudo.

Dentro do período analisado (1986-2006), o padrão espaço-temporal da OCA2 foi observado propagando-se para leste ao redor do Oceano Sul de 1983 até 2001 (Fig. 3), quando as anomalias associadas a OCA2 enfraquecem consideravelmente, não sendo mais possível detectá-la. Já para a OCA3, o padrão espaço-temporal característico foi observado propagando-se ao redor do Oceano Sul de 1981 até 1996, quando ocorre um enfraquecimento das anomalias, as quais recomeçam a fortalecer posteriormente a partir do final de 2002 (Fig. 4). Estes resultados concordam com a evolução temporal das componentes OCA2 e OCA3 já encontrada por Venegas (2003), onde o período analisado foi de 1969 a 2000 . A autora encontrou a OCA2 propagando-se ao redor do Oceano Sul de 1985 até 2000 e a OCA3 de 1978 até 1997. Ambos os resultados sugerem que periodicamente ocorre um amortecimento do sinal das componentes interanuais da OCA quando as mesmas propagam-se ao redor do Oceano sul. 


\subsection{Padrões de associação entre a Onda Circumpolar Antártica e as anomalias anuais de precipitação}

Como neste estudo as componentes OCA2 e OCA3 foram identificadas, respectivamente, nos períodos de 1983 a 2001 e de 1981 a 1996, a influência das mesmas sobre as anomalias anuais de precipitação foi analisada somente ao longo destes períodos. Além disso, como os setores Pacífico Leste e Atlântico Oeste do Oceano Sul constituem as principais regiões fontes dos sistemas meteorológicos que atuam no RS (Reboita et al., 2010), esses foram considerados para investigar a influência de ambas as componentes sobre as anomalias anuais de precipitação do RS. O setor Pacífico Leste foi aqui considerado como a região situada entre $40^{\circ} \mathrm{S}-60^{\circ} \mathrm{S}$ e entre $120^{\circ} \mathrm{W}-70^{\circ} \mathrm{W}\left(240^{\circ} \mathrm{E}-290^{\circ} \mathrm{E}\right)$. Já o setor Atlântico Oeste compreende a região situada entre $40^{\circ} \mathrm{S}-60^{\circ} \mathrm{S}$ e entre $70^{\circ} \mathrm{W}-35^{\circ} \mathrm{W}$.

\subsubsection{Padrões de Associação: Anomalias anuais de precipitação e OCA3}

Buscando determinar os padrões de associação entre o comportamento das anomalias de TSM, PNMM, UMI e VMS associadas a componente OCA3 e as anomalias anuais regionais de precipitação, a Tabela 3 foi construída. A Tabela 3 mostra as anomalias de TSM, PNMM, UMI e VMS associadas a componente OCA3 para o setor do Atlântico Oeste, para a banda de latitude entre $40^{\circ} \mathrm{S}$ e $60^{\circ} \mathrm{S}$. Uma tabela similar foi construída para o setor do Pacífico Leste (não mostrada aqui), porém nenhum padrão evidente de associação pôde ser determinado. A partir de uma análi- se da Fig. 4 e da Tabela 3 pôde-se determinar que anos com anomalias negativas de precipitação (1981, 1988, 1991, 1995, 1996) em 3 ou 4 regiões homogêneas estão associados tanto com condições simultâneas de anomalias positivas de TSM e PNMM, como com condições simultâneas de anomalias negativas de TSM e positivas de PNMM no setor Atlântico Oeste do Oceano Sul. Já anos com anomalias positivas de precipitação $(1982,1983,1984,1986,1987$, 1990, 1992, 1993, 1994) em 3 ou 4 regiões homogêneas estão associados tanto com condições simultâneas de anomalias negativas de TSM e PNMM, como com condições simultâneas de anomalias positivas de TSM e negativas de PNMM no setor Atlântico Oeste do Oceano Sul.

Os resultados encontrados neste estudo e por White e Chen (2002) sugerem que nos setores Pacífico Leste e Atlântico Oeste do Oceano Sul, o papel predominante do centro de anomalia positiva de PNMM de núcleo quente sobre a anomalia positiva de TSM leva a anomalias negativas de UMI na região. Com base neste resultado, é sugerido aqui que essas anomalias negativas (positivas) de UMI resultem em anomalias negativas (positivas) de precipitação no RS. Essas anomalias de UMI (positivas ou negativas) no setor Atlântico Oeste do Oceano Sul seriam transportadas por sistemas meteorológicos oriundos de latitudes extratropicais, como frentes frias e ciclones extratropicais, que alcançam o Estado do RS ao longo de todo o ano (Reboita et al., 2010).

Para confirmar as relações qualitativas encontradas entre os padrões de comportamento entre as anomalias de

Tabela 3 - Anomalias normalizadas de PRP, TSM, PNMM, UMI e VMS associadas a componente OCA3 para o setor do Atlântico Oeste, para a banda de latitude entre $40^{\circ} \mathrm{S} \mathrm{e} 60^{\circ} \mathrm{S}$, trajetória principal da Onda Circumpolar Antártica, para o período de 1981 a 1996. As siglas PRP01, PRP02, PRP03, PRP04 representam, respectivamente, as anomalias de precipitação das regiões homogêneas 1, 2, 3 e 4.

\begin{tabular}{|c|c|c|c|c|c|c|c|c|}
\hline \multicolumn{9}{|c|}{ OCA3 } \\
\hline ANO & PRP01 & PRP02 & PRP03 & PRP04 & TSM & PNMM & VMS & UMI \\
\hline 1981 & $-0,480$ & $-1,260$ & $-1,370$ & $-1,420$ & 0,041 & 0,353 & $-0,005$ & $5,33 \mathrm{E}-006$ \\
\hline 1982 & 0,060 & 0,480 & $-0,130$ & 0,130 & 0,130 & $-0,238$ & $-0,062$ & $3,01 \mathrm{E}-005$ \\
\hline 1983 & 0,210 & 0,260 & 1,790 & 1,700 & $-0,111$ & $-0,296$ & 0,034 & $-1,97 \mathrm{E}-005$ \\
\hline 1984 & 0,410 & 1,360 & 1,200 & 0,570 & $-0,111$ & 0,389 & 0,059 & $-2,74 \mathrm{E}-005$ \\
\hline 1985 & $-0,210$ & 0,440 & $-0,890$ & 0,130 & 0,159 & 0,241 & $-0,054$ & $2,97 \mathrm{E}-005$ \\
\hline 1986 & 0,510 & 1,740 & $-0,160$ & 0,530 & 0,100 & $-0,499$ & $-0,059$ & $2,64 \mathrm{E}-005$ \\
\hline 1987 & 0,230 & 0,660 & 1,070 & 0,690 & $-0,200$ & $-0,216$ & 0,071 & $-3,86 \mathrm{E}-005$ \\
\hline 1988 & $-1,300$ & $-0,970$ & $-0,630$ & $-0,720$ & $-0,095$ & 0,613 & 0,061 & $-2,66 \mathrm{E}-005$ \\
\hline 1989 & $-1,150$ & $-1,110$ & 0,010 & 0,030 & 0,245 & 0,171 & $-0,093$ & $4,93 \mathrm{E}-005$ \\
\hline 1990 & 0,190 & 0,630 & 0,890 & 0,940 & 0,067 & $-0,719$ & $-0,053$ & $2,14 \mathrm{E}-005$ \\
\hline 1991 & $-0,480$ & $-0,920$ & $-1,320$ & $-1,300$ & $-0,276$ & $-0,042$ & 0,111 & $-5,77 \mathrm{E}-005$ \\
\hline 1992 & 0,180 & $-0,190$ & 0,170 & 0,540 & $-0,004$ & 0,723 & 0,025 & $-7,01 \mathrm{E}-006$ \\
\hline 1993 & $-0,160$ & 0,020 & 0,050 & 0,340 & 0,250 & $-0,146$ & $-0,108$ & $5,44 \mathrm{E}-005$ \\
\hline 1994 & $-0,180$ & 0,650 & 0,740 & 0,660 & $-0,067$ & $-0,541$ & 0,012 & $-1,06 \mathrm{E}-005$ \\
\hline 1995 & $-0,460$ & $-0,800$ & $-0,890$ & $-1,390$ & $-0,154$ & 0,267 & 0,072 & $-3,48 \mathrm{E}-005$ \\
\hline 1996 & $-0,930$ & $-0,640$ & 0,380 & $-0,350$ & 0,092 & 0,217 & $-0,034$ & $1,94 \mathrm{E}-005$ \\
\hline
\end{tabular}


TSM e PNMM associadas a componente OCA3 e anomalias anuais regionais de precipitação, coeficientes de regressão e correlação foram calculados entre as anomalias de PNMM e as anomalias de precipitação das 4 regiões homogêneas (Tabela 4), para o período de 1981 a 1996. A análise foi realizada com as anomalias de PNMM, pois os resultados encontrados mostram que enquanto estas parecem ser determinantes na ocorrência de anomalias positivas ou negativas de precipitação no RS, as anomalias de TSM parecem ter um papel secundário. A partir da Tabela 4 pode ser notado que os coeficientes de regressão e correlação confirmaram a relação negativa entre as anomalias de PNMM associadas a OCA3 e as anomalias anuais regionais de precipitação, corroborando que anomalias negativas (positivas) de PNMM associadas a OCA3 no Atlântico Oeste favorecem a anomalias positivas (negativas) de precipitação no Estado do RS. Além disso, pode ser verificado que a porção Central e Noroeste (Regiões 2 e 4) do RS foi a que apresentou maiores valores de coeficientes de regressão e correlação, sugerindo que esta região é a que está sujeita a maior influência da OCA3. Relembrando os valores de RMS apresentados na Tabela 2, e combinando com os valores dos coeficientes de regressão e correlação apresentados na Tabela 4, pode-se sugerir que a maior variabilidade interanual observadas nas Regiões 2 e 4 (Tabela 2) pode estar associada a uma maior influência da OCA3 sobre os sistemas meteorológicos que mais influenciam o regime de chuvas nestas regiões. Como mencionado na seção 3.1, segundo Britto et al. (2006), na região centro-sul do Estado, os meses de inverno são os mais chuvosos, devido a freqüência de passagens dos sistemas frontais, e na região noroeste do RS, os meses da primavera são os mais chuvosos, devido a formação dos Complexos Convectivos de Mesoescala. Desde que esses sistemas meteorológicos são fortemente influenciados pelos campos de PNMM e UMI (Reboita et al., 2010), os resultados encontrados aqui mostram uma importante influência da OCA3 sobre os sistemas via, por exemplo, anomalias negativas de PNMM, e consequentemente sobre anomalias anuais positivas de precipitação nestas regiões.

Tabela 4 - Coeficientes de Regressão e Correlação entre as anomalias de PNMM do Setor Atlântico Oeste do Oceano Sul, para a banda de latitude de $40^{\circ} \mathrm{S} \mathrm{a} 60^{\circ} \mathrm{S}$ e as anomalias de precipitação das 4 regiões homogêneas, para o período de 1981 a 1996.Somente os coeficientes de regressão e correlação entre as anomalias de PNMM e as anomalias de precipitação da Região 02 foram significantes ao nível de $95 \%$.

\begin{tabular}{lcc}
\hline Regiões/Coeficientes & $\begin{array}{c}\text { Coeficiente de } \\
\text { Correlação }\end{array}$ & $\begin{array}{c}\text { Coeficiente de } \\
\text { Regressão }\end{array}$ \\
\hline Região 01 & $-0,455$ & $-0,590$ \\
Região 02 & $-0,536$ & $-1,156$ \\
Região 03 & $-0,395$ & $-0,871$ \\
Região 04 & $-0,487$ & $-1,033$ \\
\hline
\end{tabular}

Além disso, os maiores coeficientes de regressão e correlação podem ser explicados pelas sucessivas mudanças anuais das anomalias de TSM e PNMM no Oceano Sul, devido a propagação contínua para leste das anomalias de TSM, que invertem a polaridade das anomalias de PNMM a medida que se propagam, propiciando uma inversão contínua das anomalias de TSM, VMS e PNMM observadas no setor Atlântico Oeste do Oceano Sul.

Um importante resultado também foi encontrado analisando-se as anomalias de UMI sobre o sul do RS $\left(30^{\circ} \mathrm{S}\right.$ $35^{\circ} \mathrm{S}$ e $\left.300^{\circ} \mathrm{E}-305^{\circ} \mathrm{E}\right)$. A Tabela 5 mostrou que dos seis anos $(1982,1983,1984,1986,1987,1990)$ em que anomalias positivas de precipitação foram observadas nas regiões homogêneas 1 e 2, em quatro anos (com exceção de 1982 e 1984) anomalias positivas de UMI associadas a OCA3 foram observadas sobre as duas regiões homogêneas. Também foi encontrado que dos seis anos (1981, 1988, 1989, 1991, 1995 e 1996) em que anomalias negativas de precipitação foram observadas nas duas regiões 1 e 2, em quatro anos (com exceção de 1991 e 1996) anomalias negativas de UMI associadas a OCA3 foram observadas. Segundo Reboita (2008), a disponibilidade de umidade favorece a precipitação que, por consequência, libera calor latente que aquece a coluna atmosférica e induz convergência na baixa troposfera, favorecendo a ocorrência de ciclogêneses. Portanto, os resultados encontrados aqui também indicam uma influência da OCA3 nas anomalias anuais de precipitação via anomalias anuais locais de UMI.

\subsubsection{Padrões de Associação: Anomalias anuais de precipitação e OCA2}

Diferentemente da OCA3, analisando-se a Fig. 3 e a Tabela 6, não foi possível encontrar um padrão evidente de associação entre o comportamento da OCA2, ao longo do cinturão de latitude entre $40^{\circ} \mathrm{S}$ e $60^{\circ} \mathrm{S}$, e as anomalias anuais de precipitação nas quatro regiões homogêneas do RS. Entretanto, analisando-se somente as anomalias anuais locais de UMI associadas a OCA2 sobre as regiões 1 e 2 (Tabela 7), os resultados também indicaram, assim como para a OCA3, uma influência da OCA2 nas anomalias anuais de precipitação via anomalias anuais locais de UMI. Foi encontrado que dos nove anos $(1983,1984,1986,1987$, 1990, 1997, 1998, 2000 e 2001) em que anomalias positivas de precipitação foram observadas nas duas regiões, em sete anos (com exceção de 1984 e 2000) anomalias positivas de UMI associadas a OCA2 foram encontradas nas regiões homogêneas 1 e 2. Ademais, dos seis anos (1988, 1989, 1991, 1995, 1996 e 1999) em que anomalias negativas de precipitação foram observadas nas duas regiões 1 e 2, em quatro anos (com exceção de 1991 e 1996) anomalias negativas de UMI associadas a OCA2 foram observadas.

Os resultados encontrados no presente estudo indicam uma fraca influência sistemática do ramo extratropical da OCA2 nas anomalias anuais de precipitação do RS. Embora não tenha sido encontrada uma associação entre o 
Tabela 5 - Anomalias normalizadas de UMI para a região entre $30^{\circ} \mathrm{S}$ a $35^{\circ} \mathrm{S} \mathrm{e} 60^{\circ} \mathrm{W}$ a $55^{\circ} \mathrm{W}$, e associadas a componente OCA3, para o período de 1981 a 1998. As siglas PRP01, PRP02, PRP03, PRP04 representam, respectivamente, as anomalias de precipitação das regiões homogêneas 1, 2, 3 e 4.

\begin{tabular}{lccccc}
\hline \multicolumn{3}{c}{ OCA3 } & & & \\
\hline ANO & PRP01 & PRP02 & PRP03 & PRP04 & $-8,15 \mathrm{E}-005$ \\
\hline 1981 & $-0,480$ & $-1,260$ & $-1,370$ & $-1,420$ & $-1,09 \mathrm{E}-005$ \\
1982 & 0,060 & 0,480 & $-0,130$ & 0,130 & $9,72 \mathrm{E}-005$ \\
1983 & 0,210 & 0,260 & 1,790 & 1,700 & $-2,25 \mathrm{E}-005$ \\
1984 & 0,410 & 1,360 & 1,200 & 0,570 & $-1,06 \mathrm{E}-004$ \\
1985 & $-0,210$ & 0,440 & $-0,890$ & 0,130 & $4,55 \mathrm{E}-005$ \\
1986 & 0,510 & 1,740 & $-0,160$ & 0,530 & $1,19 \mathrm{E}-004$ \\
1987 & 0,230 & 0,660 & 1,070 & 0,690 & $-6,90 \mathrm{E}-005$ \\
1988 & $-1,300$ & $-0,970$ & $-0,630$ & $-0,720$ & $-1,30 \mathrm{E}-004$ \\
1989 & $-1,150$ & $-1,110$ & 0,010 & 0,030 & $1,02 \mathrm{E}-004$ \\
1990 & 0,190 & 0,630 & 0,890 & 0,940 & $-1,18 \mathrm{E}-004$ \\
1991 & $-0,480$ & $-0,920$ & $-1,320$ & 0,300 & $-1,31 \mathrm{E}-004$ \\
1992 & 0,180 & $-0,190$ & 0,170 & 0,540 & $-7,21 \mathrm{E}-005$ \\
1993 & $-0,160$ & 0,020 & 0,050 & 0,340 & $1,27 \mathrm{E}-004$ \\
1994 & $-0,180$ & 0,650 & 0,740 & $-1,390$ & $-0,350$ \\
1995 & $-0,460$ & $-0,800$ & 0,890 & $-7,84 \mathrm{E}-005$ \\
1996 & $-0,930$ & $-0,640$ & & &
\end{tabular}

Tabela 6 - Anomalias de PRP, TSM, PNMM, UMI e VMS associadas a componente OCA2 para o setor do Atlântico Oeste, para a banda de latitude entre $40^{\circ} \mathrm{S}$ e $60^{\circ} \mathrm{S}$, trajetória principal da Onda Circumpolar Antártica, para o período de 1983 a 2001. As siglas PRP01, PRP02, PRP03, PRP04 representam, respectivamente, as anomalias de precipitação das regiões homogênea 1, 2, 3 e 4.

\begin{tabular}{|c|c|c|c|c|c|c|c|c|}
\hline \multicolumn{9}{|c|}{ OCA2 } \\
\hline ANO & PRP01 & PRP02 & PRP03 & PRP04 & TSM & PNMM & VMS & UMI \\
\hline 1983 & 0,210 & 0,260 & 1,790 & 1,700 & $-0,069$ & 0,206 & 0,149 & $-7,28 \mathrm{E}-006$ \\
\hline 1984 & 0,410 & 1,360 & 1,200 & 0,570 & 0,072 & 0,309 & 0,113 & $4,53 \mathrm{E}-007$ \\
\hline 1985 & $-0,210$ & 0,440 & $-0,890$ & 0,130 & 0,118 & $-0,191$ & $-0,169$ & $9,83 \mathrm{E}-006$ \\
\hline 1986 & 0,510 & 1,740 & $-0,160$ & 0,530 & $-0,051$ & $-0,440$ & $-0,185$ & $2,36 \mathrm{E}-006$ \\
\hline 1987 & 0,230 & 0,660 & 1,070 & 0,690 & $-0,146$ & 0,062 & 0,125 & $-9,62 \mathrm{E}-006$ \\
\hline 1988 & $-1,300$ & $-0,970$ & $-0,630$ & $-0,720$ & $-0,001$ & 0,447 & 0,213 & $-5,17 \mathrm{E}-006$ \\
\hline 1989 & $-1,150$ & $-1,110$ & 0,010 & 0,030 & 0,124 & 0,114 & $-0,033$ & $6,05 \mathrm{E}-006$ \\
\hline 1990 & 0,190 & 0,630 & 0,890 & 0,940 & 0,053 & $-0,283$ & $-0,161$ & $5,77 \mathrm{E}-006$ \\
\hline 1991 & $-0,480$ & $-0,920$ & $-1,320$ & $-1,300$ & $-0,052$ & $-0,221$ & $-0,053$ & $-6,77 \mathrm{E}-007$ \\
\hline 1992 & 0,180 & $-0,190$ & 0,170 & 0,540 & $-0,069$ & 0,011 & 0,041 & $-3,15 \mathrm{E}-006$ \\
\hline 1993 & $-0,160$ & 0,020 & 0,050 & 0,340 & $-0,036$ & 0,176 & 0,081 & $-3,66 \mathrm{E}-006$ \\
\hline 1994 & $-0,180$ & 0,650 & 0,740 & 0,660 & 0,035 & 0,232 & 0,088 & $-1,55 \mathrm{E}-006$ \\
\hline 1995 & $-0,460$ & $-0,800$ & $-0,890$ & $-1,390$ & 0,095 & $-0,003$ & $-0,038$ & $4,86 \mathrm{E}-006$ \\
\hline 1996 & $-0,930$ & $-0,640$ & 0,380 & $-0,350$ & 0,026 & $-0,326$ & $-0,162$ & $5,70 \mathrm{E}-006$ \\
\hline 1997 & 0,670 & 0,630 & 0,470 & 0,980 & $-0,099$ & $-0,187$ & $-0,039$ & $-2,95 \mathrm{E}-006$ \\
\hline 1998 & 1,090 & 0,500 & $-0,110$ & 1,230 & $-0,075$ & 0,250 & 0,152 & $-7,03 \mathrm{E}-006$ \\
\hline 1999 & $-1,080$ & $-0,590$ & $-1,210$ & $-0,880$ & 0,059 & 0,279 & 0,093 & $-9,43 \mathrm{E}-008$ \\
\hline 2000 & 0,130 & 0,290 & 0,050 & 0,060 & 0,084 & $-0,089$ & $-0,083$ & $5,24 \mathrm{E}-006$ \\
\hline 2001 & 1,520 & 0,120 & 0,790 & $-0,520$ & $-0,009$ & $-0,232$ & $-0,091$ & $2,00 \mathrm{E}-006$ \\
\hline
\end{tabular}


Tabela 7 - Anomalias normalizadas de UMI para a região entre $30^{\circ} \mathrm{S}$ a $35^{\circ} \mathrm{S} \mathrm{e} 60^{\circ} \mathrm{W}$ a $55^{\circ} \mathrm{W}$, e associadas a componente OCA2, para o período de 1983 a 2001. As siglas PRP01, PRP02, PRP03, PRP04 representam, respectivamente, as anomalias de precipitação das regiões homogêneas 1, 2, 3 e 4 .

\begin{tabular}{|c|c|c|c|c|c|}
\hline \multicolumn{6}{|c|}{ OCA2 } \\
\hline ANO & PRP01 & PRP02 & PRP03 & PRP04 & UMI \\
\hline 1983 & 0,210 & 0,260 & 1,790 & 1,700 & $7,68 \mathrm{E}-005$ \\
\hline 1984 & 0,410 & 1,360 & 1,200 & 0,570 & $-2,53 \mathrm{E}-004$ \\
\hline 1985 & $-0,210$ & 0,440 & $-0,890$ & 0,130 & $-1,78 \mathrm{E}-004$ \\
\hline 1986 & 0,510 & 1,740 & $-0,160$ & 0,530 & $2,52 \mathrm{E}-004$ \\
\hline 1987 & 0,230 & 0,660 & 1,070 & 0,690 & $2,66 \mathrm{E}-004$ \\
\hline 1988 & $-1,300$ & $-0,970$ & $-0,630$ & $-0,720$ & $-1,56 \mathrm{E}-004$ \\
\hline 1989 & $-1,150$ & $-1,110$ & 0,010 & 0,030 & $-2,72 \mathrm{E}-004$ \\
\hline 1990 & 0,190 & 0,630 & 0,890 & 0,940 & $1,10 \mathrm{E}-005$ \\
\hline 1991 & $-0,480$ & $-0,920$ & $-1,320$ & $-1,300$ & $1,62 \mathrm{E}-004$ \\
\hline 1992 & 0,180 & $-0,190$ & 0,170 & 0,540 & $9,89 \mathrm{E}-005$ \\
\hline 1993 & $-0,160$ & 0,020 & 0,050 & 0,340 & $1,94 \mathrm{E}-005$ \\
\hline 1994 & $-0,180$ & 0,650 & 0,740 & 0,660 & $-1,11 \mathrm{E}-004$ \\
\hline 1995 & $-0,460$ & $-0,800$ & $-0,890$ & $-1,390$ & $-1,80 \mathrm{E}-004$ \\
\hline 1996 & $-0,930$ & $-0,640$ & 0,380 & $-0,350$ & $3,24 \mathrm{E}-005$ \\
\hline 1997 & 0,670 & 0,630 & 0,470 & 0,980 & $2,41 \mathrm{E}-004$ \\
\hline 1998 & 1,090 & 0,500 & $-0,110$ & 1,230 & $7,05 \mathrm{E}-005$ \\
\hline 1999 & $-1,080$ & $-0,590$ & $-1,210$ & $-0,880$ & $-1,89 \mathrm{E}-004$ \\
\hline 2000 & 0,130 & 0,290 & 0,050 & 0,060 & $-1,23 \mathrm{E}-004$ \\
\hline 2001 & 1,520 & 0,120 & 0,790 & $-0,520$ & $7,85 \mathrm{E}-005$ \\
\hline
\end{tabular}

comportamento das variáveis associadas a OCA2, quando as mesmas se propagam entre $40^{\circ} \mathrm{S}$ e $60^{\circ} \mathrm{S}$, e as anomalias regionais anuais de precipitação, foi constatada uma associação entre as anomalias anuais locais de UMI associadas a OCA2 e as anomalias anuais de precipitação das regiões homogêneas 1 e 2. Além disso, assim como a OCA3, a OCA2 também modifica o campo médio de TSM, PNMM e VMS, e, portanto também deve atuar sobre o comportamento de sistemas meteorológicos oriundos dos extratrópicos e que atuam no clima do RS.

\section{Conclusões}

A investigação da influência da Onda Circumpolar Antártica sobre as anomalias anuais de precipitação do RS mostrou que enquanto existe um padrão mais evidente de associação entre o comportamento da OCA3, ao longo do cinturão de latitude entre $40^{\circ} \mathrm{S}$ e $60^{\circ} \mathrm{S}$, e as anomalias anuais de precipitação nas quatro regiões homogêneas do RS, o mesmo não foi encontrado para a OCA2. Além disso, os resultados sugeriram que enquanto as anomalias de pressão ao nível médio do mar associadas a OCA3 parecem ter um papel principal na modulação das anomalias de precipitação no Rio Grande do Sul, as anomalias de temperatura da superfície do mar associadas a OCA3 parecem ter um papel secundário. Uma forte influência da OCA3 nas anomalias anuais de precipitação via anomalias anuais locais de umidade específica também foi observada. Embora não tenha sido encontrado nenhum padrão de associação entre a OCA2 e as anomalias anuais regionais de precipitação, também foi constatada uma influência local da OCA2 sobre o campo de umidade específica. Portanto além da influência extratropical, a porção subtropical da Onda Circumpolar Antártica parece também atuar sobre as anomalias anuais regionais de precipitação no sul do Rio Grande do Sul.

Assim, os achados deste estudo mostram que a Onda Circumpolar Antártica parece ter uma forte influência sobre as anomalias anuais de precipitação no Rio Grande do Sul tanto via ação remota, como modificando campos locais de umidade específica. Tal conhecimento pode significar um grande avanço na previsão climática para o estado, e assim, contribuir para uma diminuição nos prejuízos econômicos provocados pela variabilidade climática da precipitação no Rio Grande do Sul.

\section{Agradecimentos}

Os autores agradecem ao Prof. Dr. Gilberto Barbosa Diniz da FMet-UFPel, ao INMET e à FEPAGRO pelo fornecimento dos dados de precipitação. Agradecem também ao Programa de Pós-Graduação em Meteorologia/IGEO-UFRJ e a Coordenação de Aperfeiçoamento de Pessoal de Nível Superior (CAPES) pelo suporte acadêmico e financeiro durante a preparação da dissertação que serviu de base para a construção deste artigo. 


\section{Referências}

ARAÚJO, S.M.B. Estudo da variabilidade climática em regiões homogêneas de temperaturas médias do ar no Rio Grande do Sul. 54p. : il. - Dissertação (Mestrado), Pelotas, 2005.

BERLATO, M.A.; CORDEIRO, A.P.A. Variabilidade Climática e Agricultura do Rio Grande do Sul. In: FEDERACITE. As Estiagens e as Perdas na Agricultura: Fenômeno Natural ou Imprevidência?, em Esteio/RS - EXPOINTER, pág. 43, 2005.

BERLATO, M.A.; FONTANA, D.C. El Niño e La Niña: impactos no clima, na vegetação e na agricultura do Rio Grande do Sul; aplicações de previsões climáticas na agricultura. Porto Alegre: Editora da UFRGS, 110p., 2003.

BIAN, L.G.; LIN, X. Interdecadal change in the Antarctic Circumpolar Wave during 1951-2010. Advances in Atmospheric Sciences, v. 29, p. 464-470, 2012.

BRITTO, F.; BARLETTA, R.; MENDONÇA, M. Regionalização sazonal e mensal da precipitação pluvial máxima no estado do Rio Grande do Sul. Revista Brasileira de Climatologia/Associação Brasileira de Climatologia, Presidente Prudente, SP. v. 02, nº 02, p. 35-51, 2006.

BRITTO, F.P.; BARLETTA, R.; MENDONCA, M. Variabilidade espacial e temporal da precipitação pluvial no Rio Grande do Sul: influência do ENOS. Revista Brasileira de Climatologia / Associação Brasileira de Climatologia, v. 3/4, p. 37-48, 2008.

CHRISTOPH, M.; BARNETT, T.P.; ROECKNER, E. The Antarctic Circumpolar Wave in a coupled oceanatmosphere GCM. Journal of Climate, v. 11, p. 16591672. 1998.

CONNOLLEY, W.M. Long term variation of the Antarctic Circumpolar Wave. Journal of Geophysical Research, v. 107, Issue C4, p. SOV 3-1 - SOV 3-12, 2003.

FECHINE, J.A.L.; GALVÍNCIO, J.D. Agrupamento da Precipitação Mensal da Bacia Hidrográfica do Rio Brigida-PE, através da Multivariada. Revista Brasileira de Geografia Física, v. 1, p.39-46, 2008.

FONTANA, D.C.; BERLATO, M.A. Associação entre El Niño - Oscilação Sul e a produtividade do milho no ESTADO do Rio Grande do Sul. Pesquisa Agropecuária Brasileira, v. 40, fasc. 5, p. 423-432, 2005.

GUEDES, R.V. DE S.; LIMA, F.J.L.; AMANAJÁS, J.C.; BRAGA, C.C. Análise em Componentes Principais da precipitação pluvial no Estado do Piauí e Agrupamento pelo Método de Ward. Revista de Geografia (Recife), v. 27, p. 218-233, 2010.

KHAN, V.M.; KIM, I.S.; SARAIVA, J.M. A relação entre as anomalias de TSM no Hemisfério Sul com as anomalias de precipitação no Sul do Brasil. Anais do X Congresso Brasileiro de Meteorologia. SBMET, 1998.

MACHADO, J.P.; BLANK, D.M.P.; ZONTA, J.H.; Justino, F. Comportamento da precipitação e da temperatu- ra no Rio Grande do Sul baseado na análise de agrupamento. Ciência e Natura, v. 32, p. 49-63, 2010.

MANN, M.E.; PARK, J. Oscillatory Spatiotemporal Signal Detection in Climate Studies: A MultipleTaper Spectral Domain Approach. Advances in Geophysics, v. 41, p. 1-131, 1999.

PRABHU, A.; MAHAJAN, P.N.; KHALADKAR, R.M.; CHIPADE, M.D. Role of Antarctic circumpolar wave in modulating the extremes of Indian summer monsoon rainfall. Geophysical Research Letters, v. 37, Issue 14, 2010.

PRESTES, S.D.; SOUSA, A.J.S.; ROLIM, P.A.M.; SANTO, A.R.S.E.; SOUSA, J.R.A. Relações entre a Oscilação Decadal do Pacífico e a Variabilidade da precipitação em Porto Alegre RS. In: XVI Congresso Brasileiro de Meteorologia, 2010, Belém do Pará. Anais do XVI Congresso Brasileiro de Meteorologia, 2010.

REBOITA, M.S. Ciclones Extratropicais sobre o Atlântico Sul: Simulação Climática e Experimentos de Sensibilidade. Tese de Doutorado em Meteorologia, Instituto de Astronomia, Geofísica e Ciências Atmosféricas IAG-USP, 359 p., 2008.

REBOITA, M.S.; GAN, M.A.; DA ROCHA, R.P.; AMBRIZZI, T. Regimes de Precipitação na América do Sul: Uma Revisão Bibliográfica. Revista Brasileira de Meteorologia (Impresso), v. 25, p. 185-204, 2010.

SAHA,S.; MOORTHI,S.; PAN, H.-L.; WU,X.; WANG, J., et al. The NCEP Climate Forecast System Reanalysis. Bulletin of the American Meteorological Society, v. 91, p. 1015-1057, 2010.

SANTOS, D.C.; VERARDO, G.; FERRAZ, S.E.; PIVA, E.D. Avaliação das regiões homogêneas, usando o método de Análise de Agrupamento com diferentes aplicações nos dados de precipitação do Rio Grande do Sul. Ciência e Natura, Micrometeorologia - Edição Suplementar, p. 339-342, 2011.

VENEGAS, S.A. The Antarctic Circumpolar Wave: A combination of two signals?, Journal of Climate, v. 16, p. 2509-2525, 2003.

WHITE, W.B. Design of a global observing system for gyre scale upper ocean temperature variability. Progress in Oceanography. Pergamon, v. 36, p. 169-217, 1995.

WHITE, W.B.; PETERSON, R.G. An Antarctic circumpolar wave in surface pressure, wind and sea ice extent. Nature. v. 380, p. 699-702, 1996.

WHITE, W.B.; CHERRY, N.J. Influence of the Antarctic circumpolar wave upon New Zealand temperature and precipitation during autumn-winter. Journal of Climate, v. 12, p. 960-976. 1999.

WHITE, W.B. Influence of Antarctic Circumpolar Wave on Australian precipitation. Journal of Climate, v. 13, p. 2125-2141. 2000.

WHITE, W.B.; CHEN, S.C. Thermodynamic Mechanisms Responsible for the Tropospheric Response to SST 
Anomalie in the Antarctic Circumpolar Wave. Journal of Climate, v. 15, $\mathrm{n}^{\mathrm{o}}$ 18, p. 2577-2596, 2002.

WHITE, W.; ANNIS, J. Influence of the Antarctic Circumpolar Wave on El Niño and its multidecadal changes from 1950 to 2001. Journal of Geophysical Research, v. 109, Issue C6, 2004.
WHITE, W.B.; GLOERSON, P.; SIMMONDS, I. Trophospheric response of Antarctic Circumpolar Wave along the sea-ice edge around Antarctica. Journal of Climate, v. 17, p. 2765-2779, 2004.

All the contents of this journal, except where otherwise noted, is licensed under a Creative Commons Attribution License CC-BY. 